\title{
Activity-dependent immobilization of mitochondria: the role of Miro
}

\author{
F.Anne Stephenson* \\ School of Pharmacy, University of London, London, UK \\ ${ }^{*}$ Correspondence: anne.stephenson@pharmacy.ac.uk
}

\section{A commentary on}

Miro1 is a calcium sensor for glutamate receptor-dependent localization of mitochondria at synapses

by MacAskill, A. F., Rinholm, J.E., Twelvetrees, A. E., Arancibia-Carcamo, L., Muir, J., Fransson, A., Aspenstrom, P., Attwell, D., and Kittler, J. T. (2009) Neuron 61, 541-555.

It is well established that in neurones, at any one time, in the range of $13-30 \%$ of the mitochondrial population are mobile moving in both anterograde and retrograde directions utilising motor proteins and the microtubule network. It is also known that the mobility of mitochondria is affected by neuronal activity; the empirical observation being that mitochondria are immobilized by a $\mathrm{Ca}^{2+}$-dependent mechanism in response to release of neurotransmitters or post-synaptic receptor activation presumably to satisfy the high energy demands of synaptic transmission. The molecular players involved in these transitions and their modes of action are now beginning to be unravelled. Two recent papers shed new light on mechanisms of mitochondrial dynamics (MacAskill et al., 2009; Wang and Schwarz, 2009). This article is principally a commentary on the MacAskill et al. (2009) paper but the findings reported therein are discussed with respect to those reported concurrently by Wang and Schwarz (2009).

The focus of MacAskill et al. (2009) is the mitochondrial protein, Miro1, and its role on the mobility of dendritic mitochondria in primary cultures of hippocampal neurones. Miro1 (and Miro2) is an atypical RhoGTPase that is found in the outer membrane of all mitochondria. It has a short C-terminal domain that is located inside mitochondria, one transmembrane domain and within the $\mathrm{N}$-terminal region which protrudes from the mitochondria into the cell cytoplasm, it has two GTPase domains, I and II, which are separated by two EF-hand calcium binding domains, EF-hands I and II. First, MacAskill et al. (2009) showed that over-expression of green fluorescent protein (GFP)-Miro1 resulted in 20\% increase of mobile mitochondria with no change in neither their density nor average velocity. Conversely, shRNAi knock-down of Miro1 resulted in $\sim 10 \%$ decrease in the percentage of mobile mitochondria. Perfusion of GFPMirol transfected neuronal cultures with glutamate and glycine to activate glutamate receptors or activation of $\mathrm{L}$-type $\mathrm{Ca}^{2+}$ channels in the presence of extracellular $\mathrm{Ca}^{2+}$ resulted in the almost complete cessation of mitochondrial movement. Expression of a form of GFP-Miro in which the two EFhand calcium binding domains were inactivated by mutation i.e., GFP-Miro $1 \Delta \mathrm{EF}$, did not change basal mitochondrial dendritic transport but importantly, glutamateinduced arrest did not occur. Co-cultures were used to demonstrate that in the presence of perfused glutamate, immobilized mitochondria were accumulated at synapses, i.e. that there is an activity-dependent positioning of mitochondria. Finally, rather than perfusing with glutamate, local stimulation of neurones with glutamate delivered via a patch pipette resulted in the halting of mitochondrial movement within a $15 \mu \mathrm{m}$ region for $150 \mathrm{~s}$ demonstrating that synaptic activation results in the recruitment of passing mitochondria to synapses.

This series of elegant experiments utilizing molecular and physiological approaches clearly demonstrates the pivotal role of Miro1 and its $\mathrm{Ca}^{2+}$ binding domain in mitochondrial transport and mitochondrial docking at synapses. Wang and Schwarz (2009) attained a similar conclusion however the actual molecular mechanism by which this occurs is a matter of dispute between the different groups. It is known that anterograde mitochondrial transport is mediated via interaction with kinesin motor proteins. Prior to the MacAskill et al. (2009) publication, experimental evidence suggested a role for the Milton/TRAK family of kinesin adaptor proteins in mediating this association (Glater et al., 2006; Smith et al., 2006). Milton/TRAKs bind to the cargo binding domain of kinesin and have been shown to recruit kinesin to mitochondria i.e. mitochondrial (i.e. Miro1)/kinesin association was indirect. In the MacAskill study, this dogma is challenged. Firstly, they showed that introduction of a kinesin functionblocking antibody into neurones resulted in a decrease in the percentage of mobile mitochondria proving the engagement of motor proteins. Kinesin/Mirol interactions were then studied by in vitro pull down assays. In the absence of $\mathrm{Ca}^{2+}$, a direct association between the kinesin heavy chains, KIF5A, $5 \mathrm{~B}$ and 5C, and Mirol was detected. In the presence of $2 \mathrm{mM} \mathrm{Ca}^{2+}$ however, the association of Mirol with these motors was significantly reduced. Mirol $\Delta \mathrm{EF}$ was also shown to bind kinesin and this association was, as may have been predicted, $\mathrm{Ca}^{2+}$-independent. Co-immunoprecipitations and pull downs from extracts of rat brain ratified these in vitro findings. Thus it was concluded that the presence of $\mathrm{Ca}^{2+}$ inhibits the Mirol/ kinesin protein-protein interaction hence the motor is dissociated from mitochondria yielding arrested movement.

As implicated above, the study of Wang and Schwarz (2009) also addressed the mechanism of $\mathrm{Ca}^{2+}$-mediated regulation of mitochondrial motility but their findings contrasted with those reported by MacAskill et al. (2009). Here, the focus was mitochondrial movement in axons of primary cultures of hippocampal neurones. Wang and Schwarz (2009) found that at least when over-expressed, kinesin is present on all mitochondria. In the presence of Milton (the Drosophila homologue of TRAK), and Miro, kinesin became highly concentrated on mitochondria providing evidence that Milton (and presumably TRAKs) are required for the recruitment of kinesin to neuronal mitochondria. 
Similarly to MacAskill et al. (2009), axonal mitochondrial transport was measured following expression of Miro1. Elevation of intracellular $\mathrm{Ca}^{2+}$ by calcimycin addition to neuronal cultures resulted in reduced bidirectional mitochondrial transport. This decreased mobility was not apparent following expression of Mirol $\triangle \mathrm{EF}$. Under high $\mathrm{Ca}^{2+}$ conditions, there was no evidence for a release of kinesin from the mitochondria and, intriguingly, immunoprecipitations now revealed an association between kinesin (the motor domain) and Mirol. This protein-protein interaction was concomitant with an observed reduced binding of kinesin to microtubules. The interpretation of these findings by the authors is that in the absence of $\mathrm{Ca}^{2+}$, Milton/TRAKs are required to recruit kinesin to mitochondria for their transport. In the presence of elevated $\mathrm{Ca}^{2+}$ however, the motor domain of kinesin binds directly to Mirol in preference to microtubules thus the motor becomes detached from the transporting network resulting in the arrest of mitochondrial movement.

In summary, both papers and also a paper from Saotome et al. (2008), are compatible in terms of Mirol being a key player in mitochondrial transport and activity-dependent docking at synapses. However, the findings of the two groups result in the proposal of two disparate mechanisms by which this occurs. How can this be reconciled? The biochemical studies of MacAskill et al. (2009) focussed on in vitro interactions between Miro and kinesin heavy chains in the presence and absence of $\mathrm{Ca}^{2+}$ but, in the absence of Milton/TRAKs. Wang and Schwarz (2009) included Miro, kinesin heavy chain and Milton for co-immunoprecipitation assays from transfected cells, a limitation here being that the three proteins are overexpressed. Despite these caveats, it may be speculated however that $\mathrm{Ca}^{2+}$ maybe important for the stabilization of the Miro, kinesin, Milton/TRAK) complex. In the presence of high $\mathrm{Ca}^{2+}$ and in the absence of Milton, the kinesin/Mirol interaction is not stabile and it is dissociated from mitochondrial membranes. In the presence of Milton/TRAK, even in high $\mathrm{Ca}^{2+}$, kinesin remains associated with mitochondria but the mode of interaction is altered i.e. rather than interacting via the Milton/TRAK-Miro linkage, there is a switch such that the kinesin motor domain does not bind to microtubules but rather associates directly with Miro1. This suggests that both proposed mechanisms may operate; in the presence of Milton/TRAKs, regulation by $\mathrm{Ca}^{2+}$ may be a transient response whereas when Milton/TRAK is absent, the effect of high $\mathrm{Ca}^{2+}$ is more sustained due to dissociation from mitochondria. So a key question is what controls the formation of kinesin, Milton/TRAK complexes? Further, Wang and Schwarz (2009) found that manipulating Mirol expression also had effects on retrograde mitochondrial transport implying that Mirol associates with dynein. Saotome et al. (2008) reported that the effects of Miro are not limited to transport. They found that Miro plays a role in mitochondrial fusion-fission switching and $\mathrm{Ca}^{2+}$ signalling. There has been a surge of activity in this field due to proteins involved in mitochondrial transport being recently identified. Further advances that may in the long term impact on understanding the pathogenesis of neurodegenerative diseases in which defective trafficking is implicated are awaited with keen anticipation.

\section{ACKNOWLEDGMENTS}

Work in the author's laboratory is supported by the BBSRC (UK) and the Alzheimer's Research Trust (UK).

\section{REFERENCES}

Glater, E. E., Megeath, L. J., Stowers, R. S., and Schwarz, T. L. (2006). Axonal transport of mitochondria requires Milton to recruit kinesin heavy chain and is light chain independent. J. Cell Biol. 173, 545-557.

MacAskill, A. F., Rinholm, J. E., Twelvetrees, A. E., Arancibia-Carcamo, L., Muir, J., Fransson, A., Aspenstrom, P., Attwell, D., and Kittler, J. T. (2009). Mirol is a calcium sensor for glutamate receptordependent localization of mitochondria at synapses. Neuron 61, 541-555.

Saotome, M., Safiulina, D., Szabadkai, G., Das, S., Fransson, A., Aspenstrom, P., Rizzuto, R., and Hjnoczky, G. (2008). Bidirectional $\mathrm{Ca}^{2+}$-dependent control of mitochondrial dynamics by the Miro GTPase. Proc. Natl. Acad. Sci. U.S.A. 105, 20728-20733.

Smith, M. J., Pozo, K., Brickley, K., and Stephenson, F. A. (2006). Mapping the GRIF-1 binding domain of the kinesin, KIF5C, substantiates a role for GRIF-1 as an adaptor protein in the anterograde trafficking of cargoes. J. Biol. Chem. 281, 27216-27228.

Wang, X. and Schwarz, T. L. (2009). The mechanism of $\mathrm{Ca}^{2+}$-dependent regulation of kinesin-mediated mitochondrial motility. Cell 136, 163-174.

Received: 20 January 2010; accepted: 17 March 2010; published online: 13 April 2010.

Citation: Stephenson FA (2010) Activity-dependent immobilization of mitochondria: the role of Miro. Front. Mol. Neurosci. 3:9. doi: 10.3389/fnmol.2010.00009

Copyright $\left({ }^{\circ} 2010\right.$ Stephenson. This is an open-access article subject to an exclusive license agreement between the authors and the Frontiers Research Foundation, which permits unrestricted use, distribution, and reproduction in any medium, provided the original authors and source are credited. 